\title{
The spacing of streaks in unsteady turbulent wall-bounded flow
}

\author{
G. J. Brereton and J.-L. Hwang \\ Department of Mechanical Engineering and Applied Mechanics, The University of Michigan, Ann Arbor, \\ Michigan 48109
}

(Received 12 August 1993; accepted 15 February 1994)

\begin{abstract}
The spacing of streaks of low-speed fluid has been studied experimentally in a wall-bounded turbulent flow in which sinusoidal unsteadiness was superposed on an otherwise steady mainstream over a range of frequencies. The modulation of phase-conditioned streak spacing about its mean value does not follow the steady wall-flow relation of $\lambda u_{z} / \nu$ equal to a constant. Instead, it is quite accurately described by a local length scale that models the momentary. value of the total shear distortion of large eddies of the flow. This single shear-distortion length scale also correlates well with the streak spacing measured in steady wall-bounded flows and in unbounded homogeneous turbulent flow at high shear rates. The apparent generality of these results implics that the streak-spacing selection mechanism depends strongly on the strain history of large-scale coherent motions and so should be investigated in the context of the coupled straining processes of turbulence production.
\end{abstract}

\section{INTRODUCTION}

Streaniwise streaks of relatively low- and high-speed fluid are distinctive features of near-wall turbulent flow. They are believed to arise through the process of redistribution of streamwise momentum by cross-stream roll-like motions, and play a central role in the production of turbulence. Since some of the earliest observations of these motions in a turbulent boundary layer were made by Kline and Runstadler, ${ }^{1}$ many subsequent studies have confirmed them to be general features of wall-bounded turbulent flows. ${ }^{2-6}$ The more recent numerical simulations of Lee et $\mathrm{l}^{7}$ have demonstrated that very similar streaks may be formed by high shear rates alone in homogeneous turbulent shear flows, without the need for blocking by a solid boundary; the spacing of these streaks was nearly twice those of wall-bounded flows when scaled by the local shear velocity. They have also shown that the mechanisms of streak generation and evolution are reproduced by the equations of turbulent motion in the rapiddistortion approximation; thus streaks arise predominantly through linear distortions of turbulence by the mean shear rather than through nonlinear turbulent interactions. In this spirit, Landahl ${ }^{8}$ adopted an approach similar to the rapiddistortion approximation, with nonlinear effects retained for sufficient time to establish initial conditions, and demonstrated how streak-like motions evolved from certain threedimensional disturbances.

One of the most consistent features of streaks within the inner layer of turbulent wall-bounded flows is their average separation of approximately $\lambda^{+}=\lambda_{\nu} / \nu \simeq 100$ (where $\lambda$ is the average spanwise spacing of low-speed streaks and $u_{\tau}$ is the friction velocity), typically from a continuous distribution of spanwise scales ranging from 20 to $200 .^{3,4}$ This average value is in agreement with numerical simulations at low Reynolds numbers, ${ }^{9}$ as well as with the experimental studies cited above. Numerous suggestions have been offered for the existence of this preferred spanwise scale, but there is no concensus on what the true explanation is. Kawahara et al. ${ }^{10}$ recently illustrated how the long-wavelength instability of a spanwise rectilinear vortex with the background flow of a turbulent boundary layer could lead to modes that generate streamwise vorticity, the most unstable of which had a spanwise spacing of about 100 wall units. Jang et al. ${ }^{11}$ extended the work of Benney and Gustavsson ${ }^{12}$ to illustrate how oblique vertical vorticity modes, forced by vertical velocity, achieved at a preferred spanwise scale a resonance that initiated downstream rolls and hence streaks. These direct resonance explanations have been questioned by Waleffe and $\mathrm{Kim},{ }^{13}$ who claimed that the scale selection of these interactions was not significant. They proposed instead that the spacing of streaks reflected the length scale in a critical Reynolds number for the self-sustenance of a sequence of coupled processes, of which streak generation was one.

In organized unsteady flows, relatively little is known about the behavior of streaks or related coherent motions. Sarpkaya ${ }^{14}$ recently studied the initiation and evolution of low-speed streaks in a continuously oscillating flow, with no mean velocity. During the short development periods of this study, the birth of ncw streaks always took place during the deceleration phase of the cycle, without any apparent necessity for companion hairpin vortices. Hwang and Brereton ${ }^{15}$ have examined streak behavior in turbulent pipe flow with superposed unsteadiness and noted that new streaks were preferentially formed during the deceleration phase of oscillatory unsteadiness. They also studied the meandering of streaks and concluded that there was no increased organization through imposed oscillation, but that streaks meandered as in steady flow. Al $\omega^{+}>0.1$ (where $\omega^{+}=\omega \nu / u_{\tau}^{2}$ and $\omega$ is the circular frequency of unsteadiness), they found some evidence of a weak organizing effect of unsteadiness-new streaks were slightly more likely to be formed behind or around $\Delta z^{+}=100$ distant from an existing streak, than between existing streaks.

Tardu and Binder ${ }^{16}$ have addressed the related issue of burst and ejection events in fully developed turbulent channel flows with superposed unsteadiness, and noted that bursts that produce single and multiple ejections respond differently to forced oscillation at different frequencies, and that each motion might be governed by different mechanisms. Brere- 
ton and Reynolds ${ }^{17}$ studied the response of a well-developed turbulent boundary layer to superposed oscillatory shear, and concluded that many features of the unsteady response were modulations of robust turbulence-producing motions of the parent flow. Examples included the phase leads and lags between $u^{\prime} u^{\prime}$ and $v^{\prime} v^{\prime}$, resulting from superposed oscillatory forcing, which marked the directions of intercomponent energy transfer in the wall splatting of the parent flow.

Based on these observations, one might predict that the imposition of organized unsteadiness on a well-developed turbulent wall flow would modulate the streak spacing about its value in the parent flow. However, the steady wall-flow characterization of $\lambda^{+}=\lambda_{\mu_{f}} / \nu \simeq 100$ would not be expected to apply in a time-localized sense, since $u_{\tau}$ in an unsteady flow is not in phase with shear velocities away from the wall, in regions where streak presence and turbulence-producing motions are most important. Because $u_{\tau}$ represents the mean effects on wall shear of the motions of wall-bounded turbulence, should there be a related unsteady velocity scale for shear through those motions that dynamically determine streak spacing? Motivated by this questions and an interest in the coupling between streak spacing and other aspects of turbulence production, an experimental study of streak spacing in well-developed turbulent wall flows with superposed unsteadiness was undertaken. For reasons of analytical convenience, the unsteadiness was a continuous sinusoidal variation in streamwise velocity, superposed on a welldeveloped wall-bounded turbulent flow, and was applied over a broad range of frequencies of oscillation.

\section{EXPERIMENTAL APPARATUS AND MEASUREMENT TECHNIQUES}

The test facility comprised a recirculating water loop with a constant-head tank, sump, pump, and a test section of translucent pipe $(57 \mathrm{~mm}$ in diameter, 160 diameters long, assuring fully developed flow over most of the test section). Flow control was achieved by motoring a profiled sleeve around a longitudinal slot milled in a bronze section at the downstream end of the pipe. Rotational position and speed of the profiled sleeve were controlled by a DC motor and amplifier with position feedback, which operated under the control of a laboratory computer. Further details of the experimental apparatus are described by Hwang. ${ }^{18}$ The mean flow Reynolds number $\left(\operatorname{Re}_{D}\right.$ referenced to centerline velocity and pipe diameter) was approximately 11700 for steady flow and all frequencies of imposed unsteadiness, with an average burst frequency of about $1.5 \mathrm{~Hz}$ (according to the correlation of Luchik and Tiederman, ${ }^{19}$ consistent with results of hydrogen-bubble visualization and burst detection techniques, such as the variable-interval time average). Superposed oscillations were forced at up to $4 \mathrm{~Hz}$ in these experiments, with temporal variation of phase-averaged velocity always a good representation of a sine wave. The timedependent velocity at the center of the pipe was then of the form

$$
\left\langle u_{\mathrm{cl}}\right\rangle=\bar{U}_{\mathrm{cl}}+\tilde{u}_{\mathrm{cl}}=U_{0}+U_{0} A \sin (\omega t+\phi),
$$

where $U_{0}$ is the mean streamwise velocity, $A$ is the dimensionless amplitude of streamwise velocity at the pipe center,
(>) denotes an average conditioned on a particular phase of the unsteady cycle, and $\mathrm{cl}$ refers to a measurement made at the pipe centerline; the symbols ${ }^{-}$and ${ }^{\sim}$ indicate the time mean and oscillatory components, respectively. The amplitude of flow oscillation decreased with increasing frequency, consistent with the impedance characterization of unsteady pipe flow by Shemer et al. ${ }^{20}$ In this series of experiments the amplitude of oscillation at the pipe centerline varied between $18 \%$ of the mean centerline velocity at $0.25 \mathrm{~Hz}$ and $10 \%$ at $4.0 \mathrm{~Hz}$.

Phase-conditioned and time series measurements of streamwise velocity were made using a laser-Doppler anemometer with frequency shifting, and a counter, which was interfaced with a laboratory computer to allow phaseresolved measurements of the instantaneous velocity of the flow. Since highly repeatable periodic motion could be imposed by the flow-control apparatus, a phase-averaging procedure was adopted for decomposition of flow variables into mean, oscillatory, and turbulent components. ${ }^{21}$ All measurements were averaged over at least 1000 ensembles. Statistical convergence in data was assumed to have been reached when the fractional tolerance (a measure of differences in $\left\langle u^{\prime} u^{\prime}\right\rangle$ over the first and second halves of the data set, normalized by the RMS level in $\left\langle u^{\prime} u^{\prime}\right\rangle$ ) reached $0.1 \%$. For oscillation at $4.0 \mathrm{~Hz}$, the typical number of cycles required to satisfy this tolerance was around 5000 .

The relatively low Reynolds number allowed the use of hydrogen-bubble visualization to study timelines and streaklines of the unsteady flow, using a pulsed voltage across a $0.05 \mathrm{~mm}$ platinum wire in the manner outlined by Schraub et $a .^{22}$ In order to view timelines initiated a constant distance from the wall, a very thin flat strip was inserted at the surface in the manner described by Achia and Thompson. ${ }^{23}$ The strip was two diameters long and one quarter of a diameter wide, and reduced the cross-sectional area by less than $1 \%$. The leading edge of the strip was smoothly faired to the pipe surface to avoid generation of secondary flows such as separation eddies. Hydrogen bubbles were generated at a height above the strip of $y^{+} \approx 6$, where wall units are referenced to the unperturbed steady flow. This location was chosen as the position closest to the wall at which streaks could be observed clearly and for which laser-Doppler anemometer measurements of the velocity field could be made still closer (for the purposes of resolving local velocities, turbulence intensities, and estimating their gradients).

Visual information was recorded using both a high-speed video camera (Kodak Ektapro 1000) and phase-conditioned photography. The split-screen capability of the video-camera system allowed both the streak patterns and the momentary phase of the unsteady forcing (the position of the profiled sleeve) to be recorded and displayed on the same frame. A recording rate of 500 frames per second could be achieved with illumination by focused video lighting, allowing excellent temporal resolution in recordings. Streaks were counted using an unsteady implementation of the technique and identification criteria of Smith and Metzler. ${ }^{24}$ A window of spanwise extent $\Delta z^{+}=120$ was marked on a video monitor, and the number of streaks within that window was counted in successive time intervals during slow-motion replay of the 
taped visualizations. The streak patterns observed at this location were also transcribed to paper to facilitate subsequent interpretations of these data in other ways. Time intervals of $t^{+}=t \bar{u}_{\tau}^{2} / \nu \simeq 4$ were used, which allowed many observations of individual streaks, the typical lifetime of which is $t^{+} \simeq 480$ in steady flow. ${ }^{24}$ For unsteady flows with cyclic oscillation, the streak counts during these time intervals were then grouped within eight equally spaced bins per cycle.

Average streak spacings were typically computed from 1500 observations made at equal intervals during a period of $65 \mathrm{~s}$ (6500 viscous units in time). The number of observations within each of the eight bins was therefore eight times fewer. This number of observations ensured that averages were well converged and that contributions of random errors were negligible. Systematic errors in the deduction of $\lambda^{t}$ were thought most likely to arise through uncertainty in $\tilde{u}_{\tau}$ (estimated at $\pm 3 \%$ with $95 \%$ certainty), and through systematic differences from other researchers in interpreting which hydrogen-bubble palterus momentarily constituted streaks (estimated at $\pm 5 \%$ with $95 \%$ certainty). These errors combined to yield an estimated uncertainty in $\left\langle\lambda^{+}\right\rangle$and $\lambda^{+}$of $.8 \%$ at the stated confidence level.

\section{EXPERIMENTAL RESULTS}

Measurements of streak spacing and profiles of streamwise velocities $\langle u\rangle$ and turbulence levels $\left\langle u^{\prime} u^{\prime}\right\rangle$ were made in time-averaged form for steady flow, and as phaseconditioned measures for unsteady flows, over a range of frequencies of oscillation about the fully developed mean How (see Hwang ${ }^{18}$ for detailed measurements). The range of frequencies of unsteadiness of the study was chosen to extend from a slowly varying disturbance (of the time scale of the largest eddies of the flow) to the high-frequency asymptote at which a Stokes solution was matched by the oscillatory component of streamwise velocity. Oscillation frequencies were expressed in wall units of the mean flow as $\omega^{+}=\omega \nu / \bar{u}_{\tau}^{2}$, where $\bar{u}_{\tau}$ was deduced from the log-linear regions of $U$ profiles; the consistency of $\bar{u}_{\tau}$ values measured from the near-wall velocity gradient with those from loglinear regions in unsteady wall-bounded flow has been assessed by Brereton. ${ }^{25}$ The dimensionlcss frequencies of oscillation of the study were $\omega^{+}=0.01,0.02,0.04,0.06,0.08$, and 0.12 , with centerline amplitudes $A$ of $17.9 \%, 17.4 \%$, $16.5 \%, 15.7 \%, 14.4 \%$, and $12.2 \%$, respectively. The burst frequency of the mean flow, expressed as $\omega^{+}$, was approximately 0.06 .

Preliminary validation measurements of the streamwise velocity and turbulence fields were carried out under steady conditions, and were generally in excellent agreement with reference near-wall measurements. ${ }^{18}$ With the hydrogenbubble wire located a fixed distance from the wall, average streak spacings were measured for flow at $\mathrm{Re}_{D}=8000$, 10500 , and 13000 , corresponding to dimensionless wire positions of $y^{+} \approx 4,6$, and 8 , respectively. The streak counting procedure described above yielded mean streak spacings of $\lambda^{+}=105,108$, and 106 , respectively, in good agreement with the widely accepted values of $\lambda^{+}=100 \pm 20$. $^{24}$ Photographs of typical streaks observed in steady and unsteady turbulent flow are shown in Hwang and Brereton. ${ }^{15}$

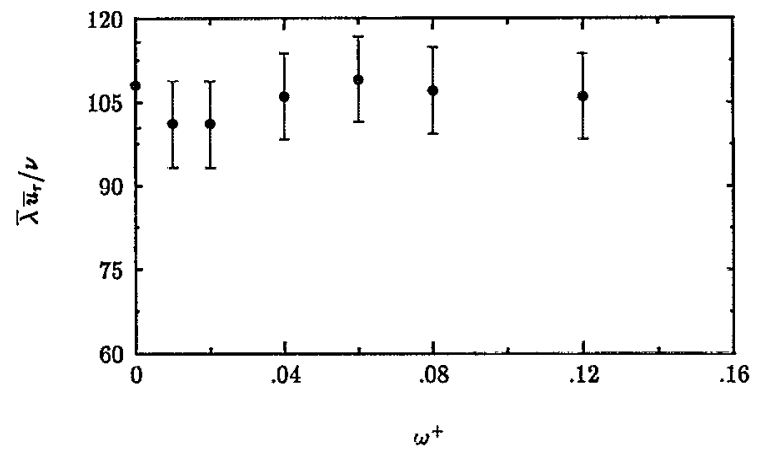

FIG. 1. Mean streak spacing at $y^{+} \approx 6$ in steady and unsteady turbulent wall-bounded flow; $\omega^{+}$is the dimensionless frequency of forced oscillation.

\section{A. Unsteady flow results}

Phase-conditioned streak spacings $\langle\lambda\rangle$ and profiles of streamwise velocity $\langle u\rangle$ and turbulence intensity $\left\langle u^{\prime} u^{\prime}\right\rangle$ were recorded for imposed sinusoidal oscillation at each of the values of $\omega^{+}$listed above. The time-average unsteady profiles of $\langle\bar{u}\rangle$ and $\overline{\left\langle u^{\prime} u^{\prime}\right\rangle}$ were scarcely distinguishable from steady-flow profiles, ${ }^{18}$ consistent with the results of many other studies of flows with imposed sinusoidal unsteadiness. The mean streak spacing $\langle\lambda\rangle \bar{u}_{\tau} / \nu$ is plotted in Fig. 1 as a function of $\omega^{+}$, and it is clear that it scarcely changes from its steady-llow value, regardless of the frequency of imposed unsteadiness. The phase-averaged streak spacing, and the streamwise velocity and turbulence intensity measured at $y^{+}=6$ are plotted together as functions of phase angle in Fig. 2, for sinusoidal oscillation at a representative frequency of $\omega^{+}=0.08$. In each of these figures, error bars indicate the uncertainty estimates given in the previous section; these uncertainties also apply to all subsequent figures in which streak spacings are plotted.

The streak spacing was strongly modulated about its mean value, and shared a similar phase dependence to $\left\langle u^{\prime} u^{\prime}\right\rangle$. The phase-averaged streak separation was also roughly in phase with the local turbulence intensity at other frequencies of this study, and this approximate phase align-

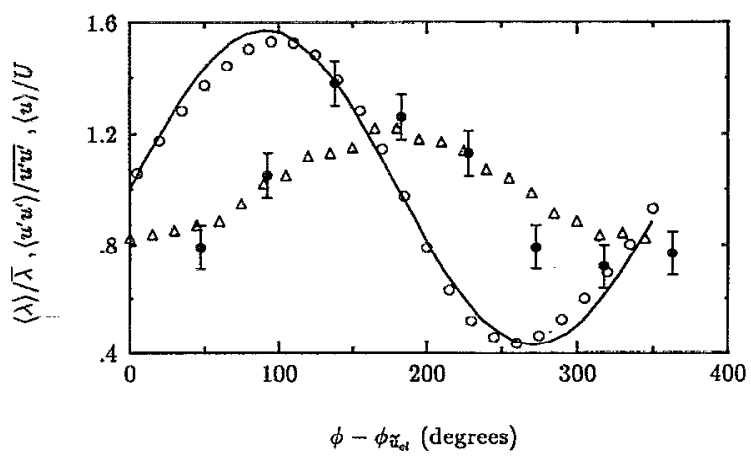

FIG. 2. Phase-averaged variation of streamwise velocity, turbulence intensity, and streak spacing in unsteady flow;,$\langle\lambda\rangle / \lambda^{\prime} ; \Delta,\left\langle u^{\prime} u^{\prime}\right\rangle / \overline{u^{\prime} u^{\prime}} ; \mathrm{O}$, $\langle u\rangle / U_{;}-$, sine wave fitted to $\langle u\rangle$ data. Measurements were made at $y^{+}=8$, $\omega^{+}=0.08$. 


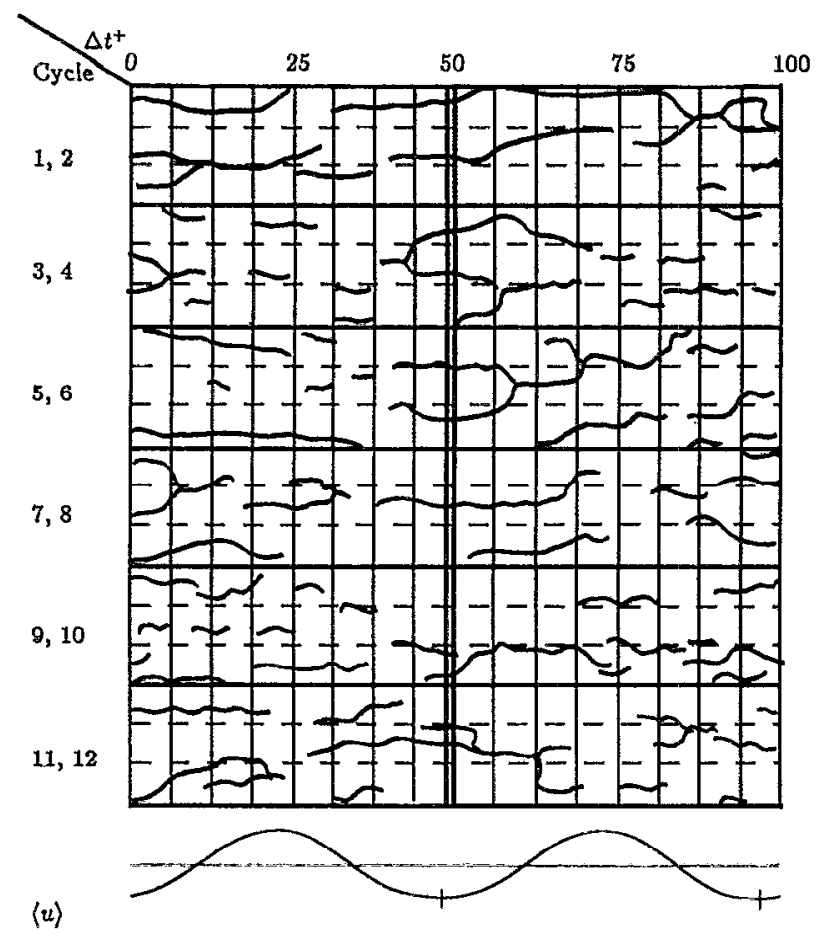

FIG. 3. Time series of streak position just downstream of a hydrogen-bubble wire at $y^{+}=6$, for 12 continuous cycles of unsteady flow at $\omega^{+}=0.12$. The reference sine wave describes the local velocity.

ment is described in detail later. Video footage ${ }^{18}$ demonstrated clearly that the initiation of new streaks was strongly organized to favor the deceleration phase of $\langle u\rangle$, when the flow is believed to be most susceptible to instability. This observation is consistent with that of Sarpkaya ${ }^{14}$ for oscillatory flow with no mean. Tardu and Binder ${ }^{16}$ have notcd a similar organization in bursting activity during the deceleration phase. The insensitivity of mean burst frequencies and streak spacing to frequency of imposed unsteadiness implies that the bursting, ejection, and streak-formation processes of turbulence production dynamically adapt to oscillatory forcing, yet are sufficiently resilient to preserve the mean character of the unforced parent flow.

A representative record of the streak positions observed at a fixed location $\left(y^{+}=6\right)$ is shown in Fig. 3 , for 12 cycles of oscillation at $\omega^{+}=0.12$, aligned with a reference sine wave fitted to the local phase-averaged streamwise velocity. In this figure, the spanwise extent of the observed field is $\Delta z^{+} \simeq 150$ with subdivisions of $\Delta z^{+} \simeq 50$. From this transcription of the video recording, a number of interesting observations may be made. First, the hydrogen-bubble wire intercepts (and marks with tracer bubbles) solitary streaks and streaks that undergo bifurcation and coalescence. Similar observations have been made by Sarpkaya ${ }^{26}$ in oscillatory-flow experiments, in which there was no mean velocity. Second, the number of observed streaks is greatest (and their average spacing smallest) toward the end of the deceleration phase of the cycle, as discussed above in reference to Fig. 2. Third, the most likely phase for the signatures of previously marked streaks to disappear is close to that of the maximum local phase-averaged velocity. Similar observations were made

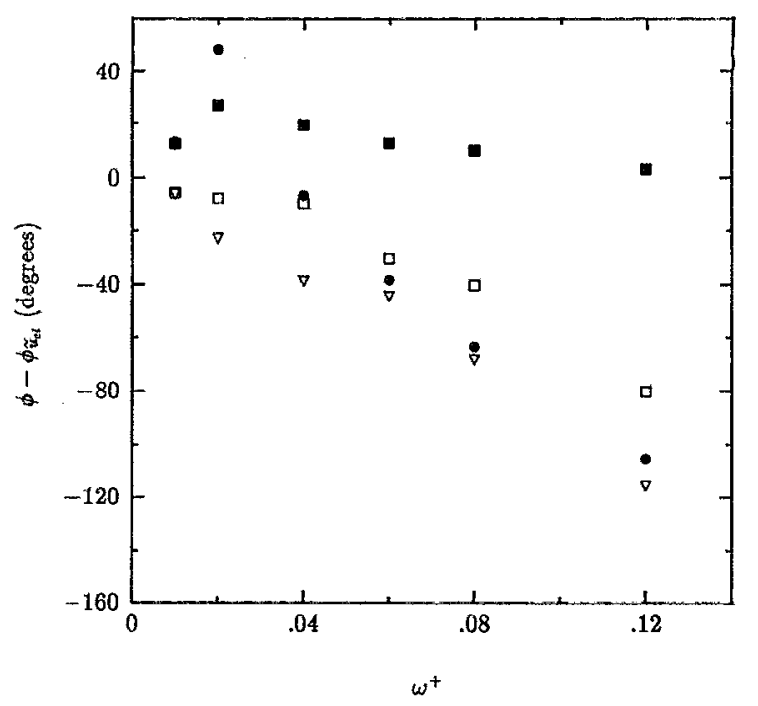

FIG. 4. Phase lead of $\langle\lambda\rangle,\left\langle u^{\prime} u^{\prime}\right\rangle$ and $\partial\langle u\rangle / \partial y$, over $\left\langle u_{\mathrm{cl}}\right\rangle$ close to the wall; -, $\phi_{\text {streak }}-\phi_{u_{\mathrm{cl}}} ; \square, \phi_{u^{\prime} u^{\prime}}-\phi_{u_{u_{\mathrm{el}}}}$ at $y^{+}=6 ; \nabla, \phi_{u^{\prime}} u^{\prime}$ at $y^{+}=8 ; \boldsymbol{\square}, \phi_{d t u / d y}$ $-\phi_{u_{\mathrm{ct}}}$ at $y^{+}=8$.

from a time series of streak position at other frequencies of unsteadiness. In the case of reciprocating flow, Sarpkaya ${ }^{26}$ observed a tendency for streaks to evolve into $\Lambda$-shaped vortical structures, which were lifted up, stretched, and then lost the coherence of their signatures. This sequence of events took place regularly in the acceleration phase, and it is conceivable that a similar series of events might lead to the termination of the streak signatures observed in this experiment. Unfortunately, the resolution of the visualization technique used in this study did not allow this issue to be addressed. Thus, while it is plausible that a mechanism for destruction of streaks exists, it must be borne in mind that an organized elevation change of the streaks, or the enhancement of diffusion during periods of strongest advcction, might also terminate observation of a streak's signature without necessitating any destruction. Because the average reported lifetime of streaks in steady-flow studies is roughly $t^{+} \simeq 480,{ }^{24}$ and signatures of the same streak in Fig. 3 may only be observed continuously at a fixed point for considerably shorter periods, it is likely that streak lifting and bubble diffusion are responsible for the termination of many of the signatures.

\section{B. Phase dependence of streak spacings}

The phase relation between $\langle\lambda\rangle,\left\langle u^{\prime} u^{\prime}\right\rangle$, and $\partial\langle u\rangle / \partial y$ is shown in Fig. 4 , in which the phase lead of the first Fourier mode of these quantities over the centerline velocity is plotted against the frequency of forced oscillation. The timelines from which the streak spacings were measured were initiated at $y^{+} \simeq 6$, while the phase of $\left\langle u^{\prime} u^{\prime}\right\rangle$ is shown for measurements made at $y^{+} \simeq 6$ and 8 . With the exception of a single data point at $\omega^{r}=0.02$, the agreement between the phase of $\langle\lambda\rangle$ and that of $\left\langle u^{\prime} u^{\prime}\right\rangle$ at $y^{\dagger}=6$ is extremely good. In a time-dependent sense, an increase in the phase-averaged streak spacing generally signifies an increase in the level of $u^{\prime} u^{\prime}$, as shown in Fig. 2 . This variation is the direct opposite 
of the behavior one would anticipate from a quasisteady extension of the steady-flow correlation: $\lambda u_{t} / \nu \approx 100$. Close to the wall, $\overline{u^{\prime} u^{\prime}} / u_{\tau}^{2}$ is a function of $y^{+}$that grows with increasing $y^{+}$. Therefore, at a fixed $y$ position, $y^{+}$and $\overline{u^{\prime} u^{\prime}}$ increase with increasing $u_{\tau}$, and a decrease in $\lambda$ would he required to preserve the steady-flow relation for dimensionless streak spacing. Thus, the correlation $\lambda u_{\tau} / \nu \simeq 100$ may have no relevance beyond steady wall-bounded flows, which is consistent with its inability to describe streak spacing in unbounded homogeneous turbulent shear flow.?

The data of Fig. 4 also show how the phases of $\left\langle u^{\prime} u^{\prime}\right\rangle$ and $\langle\lambda\rangle$ are related to the organized shear $\partial\langle u\rangle / \partial y$. They are deduced from Fourier decomposition of data. For as few as eight data points per cycle, the phase of $\langle\lambda\rangle$ is thought to be no more accurate than $\pm 20^{\circ}$. In the near-wall region, the lag in phase of $\left\langle u^{\prime} u^{\prime}\right\rangle$ behind $\partial\langle u\rangle / \partial y$ grows with $\omega^{+}$from its quasisteady value of zero toward an asymptotic highfrequency value of $90^{\circ}$, when $\partial\left\langle u^{\prime} u^{\prime}\right\rangle / \partial t$ is essentially in balance with $-\overline{u^{\prime} v^{\prime}} \partial\langle u\rangle / \partial y$ in the phase-conditioned Reynolds-stress equation. ${ }^{17}$ This orderly variation suggests that the unsteady shear and streak spacing share some relation, though its form is not obvious from these data. To explore possible dependences of streak spacing on organized shear, it is useful to approximate the temporal variation of the shear and velocity fields with the analytical solution to Stokes' second problem, for the boundary conditions of a superposed oscillatory mainstream bounded by a stationary wall. This approximation (which ignores Reynolds stress) is lent justification by the experimental data and analytical solutions plotted in Fig. 5, in which the phases and amplitudes of measured values of $\bar{u}$ are in reasonably good agreement with the Stokes solutions. Discrepancies are within $5^{\circ}$ in phase and typically less than $5 \%$ of the mainstream amplitude of oscillation, and these are no worse than the uncertainties in the measurement of $\langle\lambda\rangle$.

\section{Amplitude dependence of streak spacings}

Phase-averaged streak spacings and turbulence intensities measured at $y^{+} \simeq 6$ are plotted as functions of phase angle in Fig. 6, for sinusoidal oscillation over a range of frequencies. Although the streak spacings and turbulence intensities are quite well aligned in phase, the amplitudes of streak spacing scarcely change, while the amplitudes of $\left\langle u^{\prime} u^{\prime}\right\rangle$, and so the velocity scale $\sqrt{\left\langle u^{\prime} u^{\prime}\right\rangle}$, decrease monotonically with increasing frequency. These data for velocities and turbulence intensities are consistent with the results of numerous other unsteady-flow experiments. ${ }^{15-18}$ The streak spacing and local turbulence intensity are obviously coupled through their association with turbulence production. However, the different dependencies of their amplitudes on $\omega^{+}$ indicate that one cannot simply form a length scale from the local momentary velocity scale $\sqrt{\left\langle u^{\prime} u^{\prime}\right\rangle}$ and $\nu$, and expect it to correlate with $\langle\lambda\rangle$.

The variation of the shear amplitude may be approximated from a Cartesian-coordinate Stokes solution, and takes the form

$$
\frac{\partial \hat{u}^{+}}{\partial y^{+}}=\hat{u}_{\mathrm{cl}}^{+} \sqrt{\omega^{+}} e^{-y^{+} \sqrt{\omega^{+} / 2}} .
$$

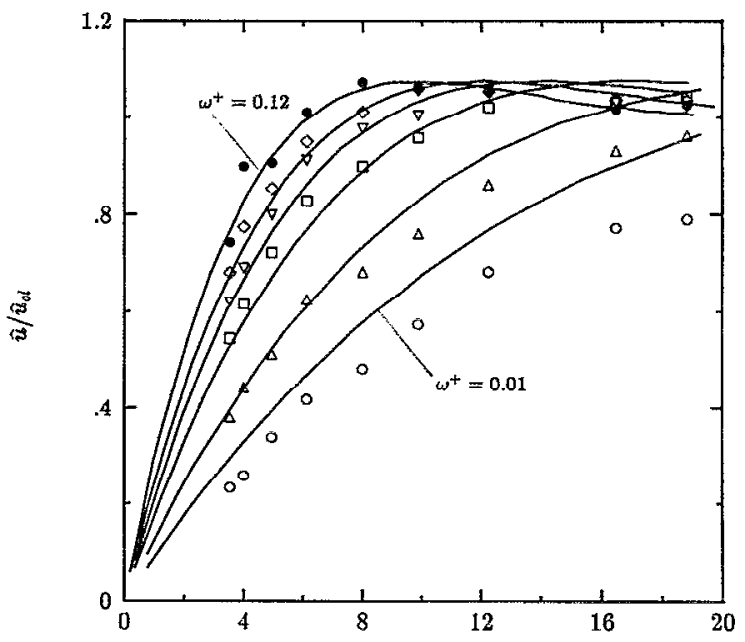

(a)
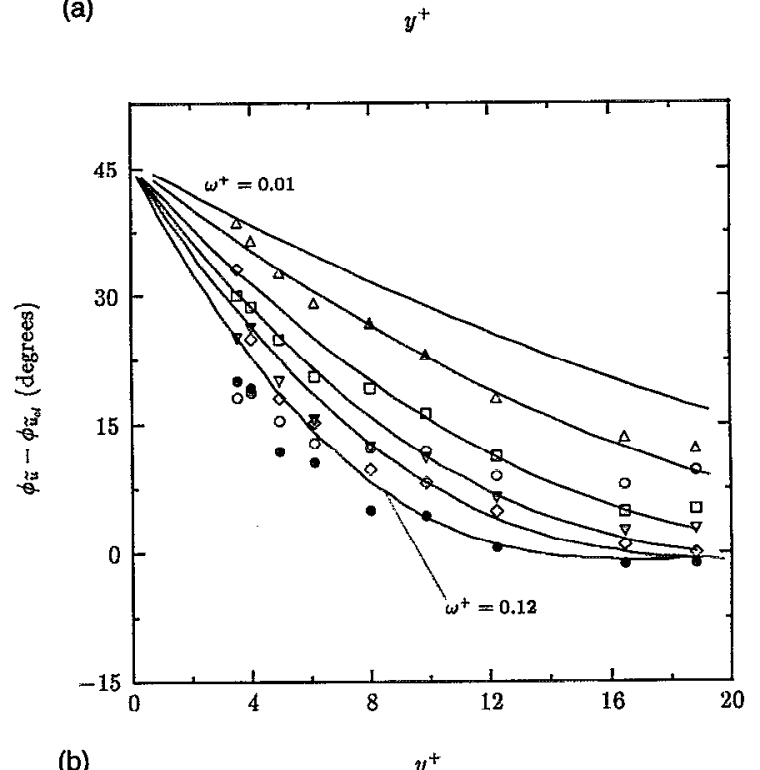

FIG. 5. (a) Near-wall profiles of amplitudes of streamwise velocity and the corresponding Stokes solutions; $O, \omega^{+}=0.01 ; \Delta, \omega^{+}=0.02 ; \square, \omega^{+}=0.04$; $\nabla, \omega^{1}=0.06 ; 0, \omega^{-1}=0.08 ; 0, \omega^{+}=0.12 ;-$, Stokes solutions for each frequency. (b) Near-wall profiles of the phase of streamwise velocity and the corresponding Stokes solutions; $O, \omega^{+}=0.01 ; \Delta, \omega^{+}=0.02 ; \square, \omega^{+}=0.04$; $\nabla, \omega^{+}=0.06 ; 0, \omega^{+}=0.08 ; \theta, \omega^{+}=0.12 ;-$, Stokes solutions for each frequency.

In this expression, $\hat{u}$ denotes the Fourier amplitude of the fundamental mode of $\langle u\rangle$, and ${ }^{+}$implies the use of wall units referenced to $u_{\tau}$ of the mean flow. This analytical result is in close agreement with the experimental data of Hwang, ${ }^{18}$ as illustrated in Fig. 5.

At a fixed amplitude of centerline oscillation and a given wall-normal position, the amplitude of $\partial\langle u\rangle / \partial y$ scales with $\sqrt{\omega^{+}} e^{-y^{+} \sqrt{\omega^{+} / 2}}$, and so peaks before decreasing with increasing $\omega^{+}$. This trend imitates the variation in amplitude of $\langle\lambda\rangle$, which can be discerned in Fig. 6. However, since $\langle\lambda\rangle$ and $\partial\langle u\rangle / \partial y$ are in phase at low values of $\omega^{+}$and around $90^{\circ}$ out of phase at the highest measured values of $\omega^{+}$, a simple streak-spacing correlation such as $\langle\lambda\rangle \sqrt{\partial\langle u\rangle / \partial y\rangle / \nu}$, cannot follow the phase of the experimental data. Based on these 


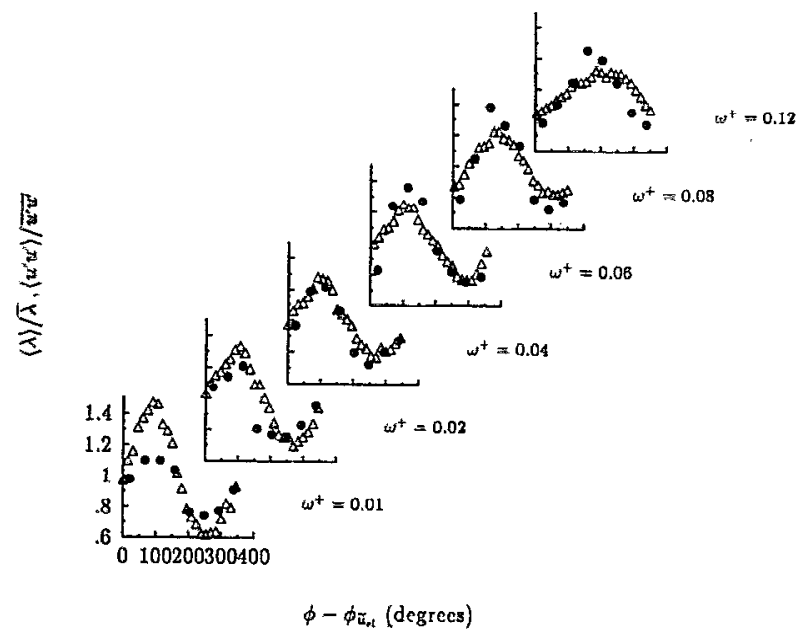

FIG. 6. Phase-conditioned variation of streak spacing and turbulence intensity;,$\langle\lambda\rangle / \bar{\lambda} ; \Delta,\left\langle u^{\prime} u^{\prime}\right\rangle / u^{\top} u^{\prime}$; measurements made at $y^{+}=6$.

phase and amplitude results, simple momentary length scales, such as $\nu /\langle u\rangle, \nu / \sqrt{\left\langle u^{r} u^{\top}\right\rangle}$, and $\sqrt{\nu /(\partial\langle u\rangle / \partial y)}$ are inadequate for correlating $\langle\lambda\rangle$. Instead, a new length scale that incorporates the average cumulative distortion of the local turbulent motions at any instant is proposed and developed in the following section.

\section{DISTORTION LENGTH SCALE FOR UNSTEADY TURBULENT SHEAR FLOW}

Distortion of the most energetic motions of turbulent shear flows has been characterized by, amongst others, Townsend and co-workers ${ }^{28-30}$ using the rapid-distortion theory. Although the formal assumptions of rapid-distortion theory restrict its use to flows in which distortion by mean shear is much greater than the fluctuating strain rates of large eddies, Townsend ${ }^{28}$ and Maxey ${ }^{31}$ have shown how the structure of shear flows undergoing slower distortion is well characterized by an effective shear-strain parameter. In a simple shear flow, the local rapid-distortion strain,

$$
\alpha(\mathbf{x}, t)=\int_{t_{0}}^{t} \frac{\partial U\left(\mathbf{x}, t^{\prime}\right)}{\partial y} d t^{\prime} \quad \text { or } \frac{\partial \alpha}{\partial t}=\frac{\partial U}{\partial y},
$$

may be combined with the strain relation for equilibrium flow:

$$
\alpha=T \frac{\partial U}{\partial y},
$$

with $T$ as a slow-distortion (large eddy) time scale, to form a generalized relation that holds for both asymptotically rapid and slow strains ${ }^{31}$ or

$$
\frac{\partial \alpha}{\partial t}=\frac{\partial U}{\partial y}-\frac{\alpha}{T} \text {. }
$$

The average effective distortion undergone by the large-scale motions of the flow may be estimated as the value of $a$ necessary to produce the same local turbulence structure (in terms of ratios of turbulent stresses) under continuous uniform-shear distortion from an initial state. Maxey ${ }^{31}$ has shown that good agreement is found between experimental and predicted values of turbulent velocity moments in fully developed pipe and channel flows, when the initial state is taken as that of approximately axisymmetric turbulence at the centerline. A more physical interpretation is that the history of large-scale coherent motions is one of entrainment from the outer regions of the flow (i.e., through sweeps) to the location of interest, as if under a continuous sequence of locally uniform shears.

Since the local shear-strain parameter $\alpha$ carries information on the turbulence structure, the scaling of $\alpha$ by a local viscous length scale forms a local shear-distortion length scale. The strong association between streak generation and shear $S$ suggests the viscous length scale: $\sqrt{\nu / S}$. It simplifies to $\sqrt{\nu /(\partial U / \partial y)}$ in steady parallel flows and to $\nu / u_{\tau}$ at a wall. In unsteady flows, the shear-distortion length scale is then $\langle\alpha\rangle \sqrt{\nu /\langle S\rangle}$. The rapid-distortion basis of this length scale, and the capability of the rapid-distortion approximation to describe the essential dynamics of streak formation, ${ }^{7}$ suggest a general unsteady scaling of streak spacing on a momentary shear-distortion length scale. Therefore, $\langle\lambda\rangle \sqrt{\langle S\rangle / \nu} /\langle\alpha\rangle$ is proposed as a characteristic dimensionless streak spacing to relate the distortion of large-scale eddies to the scalc selection of streak separation.

The strain parameter $\langle\alpha\rangle$ may be estimated in unsteady flow by rewriting (5) in its phase-conditioned form:

$$
\frac{\partial\langle\alpha\rangle}{\partial t}=\frac{\partial\langle U\rangle}{\partial y}-\frac{\langle\alpha\rangle}{T} .
$$

In the case of sinusoidal oscillation superposed on a parent mean flow,

$$
\langle\alpha\rangle=\bar{\alpha}+\hat{\alpha} e^{i \omega t} \quad \text { and } \quad\langle u\rangle=U+\hat{u} e^{i \omega t},
$$

where - is now a complex amplitude. Substitution into (6) and reversion to wall units of the parent flow yield the strain amplitude and phase lead (relative to the velocity field):

$$
|\hat{\alpha}|=\frac{\partial \hat{u}^{+}}{\partial y^{+}} \frac{T^{+}}{\sqrt{1+\left(\omega^{+} T^{+}\right)^{2}}} ; \quad \phi_{\alpha}=\arctan \left(-\omega^{+} T^{+}\right) .
$$

The strain amplitude may then be rewritten using the shear amplitude from (2) as

$$
|\hat{\alpha}|=\hat{u}_{\mathrm{cl}}^{+} \sqrt{\omega^{+}} e^{-y^{+} \sqrt{\omega^{+} / 2}} \frac{T^{+}}{\sqrt{1+\left(\omega^{+} T^{+}\right)^{2}}} .
$$

By referencing the large-eddy time scale $T^{+}$to the parent flow using (4), $T^{+} \approx \bar{\alpha}$ close to the wall. Maxey ${ }^{31}$ has found that $\bar{\alpha}$ takes the value of approximately 3.5 as the wall is approached in parallel flows. In the vicinity of $y^{+}=6$, the normalized amplitudes $|\hat{\alpha}| / \bar{\alpha}$ and $|\hat{\lambda}| / \lambda^{-}$are in good agreement. This result is shown in Fig. 7 , in which the streak separation data of Fig. 6 are superposed on plots of $\langle\alpha\rangle$. The agreement is comparable if $\langle\alpha\rangle$ is computed at $y^{+}=5$ or 7 . There are discrepancies in phase for the results at $\omega^{+}=0.12$, which may represent the limits in this single length scale theory. Finally, the streak separation data of Fig. 6 are replotted in normalized form as $\langle\lambda\rangle \sqrt{\langle S\rangle / \nu} /\langle\alpha\rangle$ in Fig. 8 as a function of phase angle. The collapse of phase-averaged 


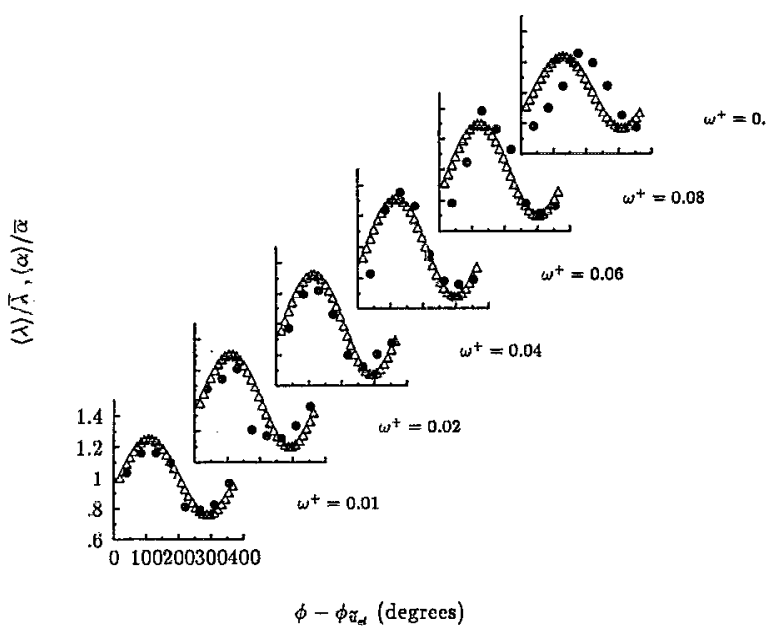

FIG. 7. Phase-conditioned variation of streak spacing and the distortion strain parameter; $0,\langle\lambda\rangle / \bar{\lambda} ; \Delta,\langle\alpha\rangle / \bar{\alpha}$; measurements made at $y^{+}=6$.

streak spacing as $\langle\lambda\rangle \sqrt{\langle S\rangle / \nu} /\langle\alpha\rangle$ with a mean of 29.2 and a standard deviation of 5.5 (from a sample of 56 data) is quite reasonable, given the experimental uncertainty, the approximations made, and the characterization by a single length scale. It indicates clearly the significance of the momentary value of this length scale in streak spacing selection.

It is useful to expand $\langle\lambda\rangle \sqrt{\langle S\rangle / \nu} /\langle\alpha\rangle$ by replacing $\langle S\rangle$ and $\langle\alpha\rangle$ by $\bar{S}+\tilde{S}$ and $\bar{\alpha}+\tilde{\alpha}$ and using (2) and (9). When this expansion is made for small variations of $\langle S\rangle$ and $\langle\alpha\rangle$ about their means, the contributions of $\hat{S}$ and $\hat{\alpha}$ to $\langle\lambda\rangle \sqrt{\langle S\rangle / \nu} /\langle\alpha\rangle$ are in the ratio

$$
\frac{\hat{\alpha} / \bar{\alpha}}{\hat{S} /(2 \bar{S})}=\frac{2 T^{+}}{\sqrt{1+\left(\omega^{+} T^{+}\right)^{2}}} \simeq 7,
$$

where the approximate value of 7 holds for all frequencies of unsteadiness of this study. Thus, the effect of unsteady straining is much more significant to changes in streak spacing than that of unstcady shcar, and $\sqrt{\langle S\rangle \nu}$ can bc approximated by the mean shear velocity of the parent flow $\bar{u}_{\tau}$.

Nearly all related experimental studies of unsteady wallbounded turbulence have been at frequencies in the range

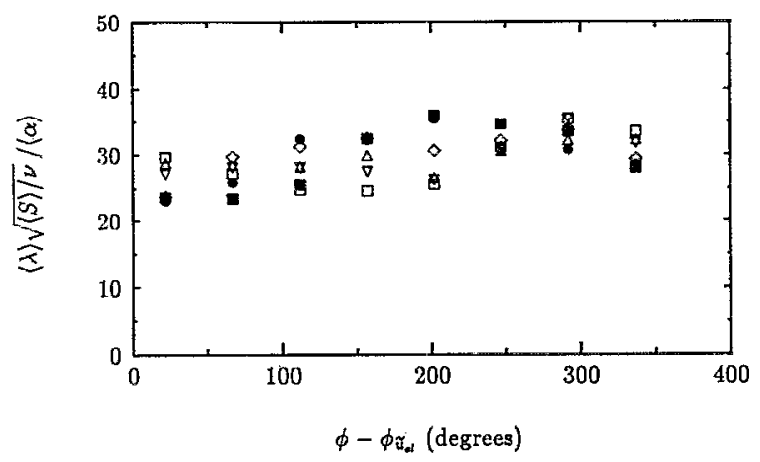

FIG. 8. Streak spacing normalized by the local momentary distortion-strain length scale; $\triangle, \omega^{+}=0.01 ; \square, \omega^{+}=0.02 ; \nabla, \omega^{+}=0.04 ; \diamond, \omega^{+}=0.06 ; \bullet$, $\omega^{+}=0.08 ; \quad B, \omega^{=}=0.12$. $0.001<\omega^{+}<0.2$. When $T^{+}$is taken as its parallel-flow nearwall value of about 3.5 , the relative change in the total strain of eddies, $|\hat{\alpha}| / \hat{\alpha}$, is typically more than three times larger than that of the local shear, $|\hat{S}| / \bar{S}$. Thus, quantities that are dependent on the momentary distortion strain $\alpha$, such as ratios of Reynolds stresses and components of turbulent kinetic energy, should respond more strongly to organized unsteadiness than those that are primarily determined by the local shear. For example, in unsteady turbulent boundary-layer flow, the production of $\widetilde{u}^{\prime} u^{\prime}$ is through interactions between mean and oscillating components of Reynolds stress and shear, via the terms $-\overline{u^{\prime} v^{\prime}} \partial \tilde{u} / \partial y$ and $-\widetilde{u^{\prime} v^{\prime}} \partial U / \partial y$. Measurements of Brereton and Reynolds ${ }^{17}$ showed that the former term exceeded the latter at all frequencies studied, though no explanation was offered. If $\langle\alpha\rangle$ is taken as proportional to the stress ratio $\left\langle-u^{\prime} v^{\prime}\right\rangle /\left\langle u^{\prime} u^{\prime}\right\rangle$, with a constant of proportionality at approach to the wall of about 4 in steady flow (see Maxey, ${ }^{31}$ Fig. 6), then

$$
\begin{aligned}
& |\hat{\alpha}|=|\langle\alpha\rangle-\bar{\alpha}| \simeq 4\left|\frac{\left\langle-u^{\prime} v^{\prime}\right\rangle}{\left\langle u^{\prime} u^{\prime}\right\rangle}-\frac{-\overline{u^{\prime} v^{\prime}}}{\overline{u^{\prime} u^{\prime}}}\right| \\
& \approx 4\left|\frac{-\widehat{u^{\prime} v^{\prime}} /\left(-\overline{u^{\prime} v^{\prime}}\right)}{\widehat{u^{\prime} u^{\prime} / \overline{u^{\prime} u^{\prime}}}}\right|,
\end{aligned}
$$

and substitution from (8) leads to

$$
\frac{-\overline{u^{\prime} v^{\prime}}(\partial \hat{u} / \partial y)}{\left|\widehat{u^{\prime} v^{\prime}}\right|(\partial U / \partial y)} \approx \frac{4}{\partial u^{+} / \partial y^{+}} \frac{\sqrt{1+\left(\omega^{+} T^{+}\right)^{2}}}{T^{+}} \frac{\overline{u^{\prime} u^{\prime}}}{\left|\widehat{u^{\prime} u^{\prime}}\right|} \text {. }
$$

For $\partial u^{+} / \partial y^{+} \leqslant 1$ and $T^{+} \approx 3.5$ at the wall, and $\widehat{u^{\prime} u^{\prime}}$ necessarily less than $\overline{u^{\prime} u^{\prime}}$, this expression exceeds unity for all values of $\omega^{+}$in the range given above. Thus, the experimental results ${ }^{17}$ that $-\overline{u^{\prime} v^{\prime}} \partial \tilde{u} / \partial y>-\widetilde{u^{\prime} v^{\prime}} \partial U / \partial y$ in unsteady wall-bounded are consistent with this rapid-distortion description of the importance of unsteady straining relative to unsteady shear. Further details of these rapid-distortion characterizations of turbulence in unsteady flows will be reported in a subsequent paper.

In general, it is difficult to predict the relative strength of response of unsteady turbulence phenomena according to their dependence on unsteady strain and shear, because it is rare that they are strongly associated with one effect but not the other. However, when unsteadiness is superposed on established turbulent flows, components of the Reynolds-stress tensor, ${ }^{17}$ burst frequencies ${ }^{16}$ wall-shear stress, ${ }^{16}$ and turbulence production terms ${ }^{17}$ are all modulated about their parent-flow values, rather than responding as independent facets of an oscillatory flow field alone. Thus, the turbulent processes of the parent flow are likely to be resilient to organized deformation through effects of both unsteady shearing and straining.

\section{DISCUSSION AND CONCLUDING REMARKS}

The generality of the scaling proposed for streak spacing in developed flows with superposed unsteadiness may also be tested against data for steady wall-bounded flow and un- 
bounded homogeneous shear flow. While it may be inferred from the unsteady data of this study (Fig. 8) that there is a momentary dimensionless streak spacing of

$$
\frac{\langle\lambda\rangle}{\langle\alpha\rangle} \sqrt{\frac{\langle S\rangle}{\nu}}=\frac{\langle\lambda\rangle u_{\tau}}{\langle\alpha\rangle \nu} \approx 29
$$

the equivalent steady-flow form of this correlation, with an effective near-wall value of $\alpha=3.5$, is

$$
\frac{\lambda u_{\tau}}{\alpha \nu} \simeq \frac{100 \pm 20}{3.5}=29 \pm 6
$$

which is in excellent agreement with the unsteady relation in (13). Moreover, $\alpha$ decreases with increasing distance from the wall in steady flows, ${ }^{31}$ consistent with experimental measurements ${ }^{24,32}$ of $\lambda$ growing with $y^{+}$and rcsults of the rapid-distortion-theory computations of Lee and Hunt. ${ }^{33}$

A further test can be made against data from the highshear homogeneous turbulence simulations of Lee et al. ${ }^{7,27}$ Their correlation of $\lambda \sqrt{(\partial U / \partial y) / \nu} \approx 200$ in a shear flow for which $\alpha=\int_{0}^{t}\left(\partial U\left(t^{\prime}\right) / \partial y\right) d t^{\prime}=8$ reduces to one of $\lambda \sqrt{(\partial U / \partial y) / \nu} / \alpha \simeq 25$, which is within the uncertainty of (14) and within one standard deviation of the result of (13). These tests indicate that the relation may have considerable generality beyond the flows considered, and that the steady wall-flow result of $\lambda u_{\tau} / \nu \simeq 100$ is merely a special case of the $\langle\lambda\rangle \sqrt{\langle S\rangle / \nu} /\langle\alpha\rangle$ correlation, in which the effective strain of energetic motions tends to around 3.5 at approach to a wall. It is not clear that this approach is relevant to undeveloped unsteady flows, such as reciprocating flows. ${ }^{14}$ In these flows, streaks periodically appear and disappear, turbulence may not be sustained throughout a cycle, and in the absence of a mean flow the slow-distortion time scale must also evolve with time to limit the growth of $\alpha$ in (6). These added complexities require considerably more than a single length scale description and are beyond the scope of this paper.

The results given above indicate that a local distortion strain and a shear length scale carry sufficient information to describe the average spacing of streaks in several developed turbulent flows. The apparent success of this approach has interesting implications for studies of turbulent structures believed to be connected with streaks and their spacing. While different boundary conditions can presumably favor different kinds of structures, the results of this study suggest that a given structure's identification with shear and the average local distortion strain determines its importance in selecting the spacing of streaks.

The local distortion strain incorporates strain-history effects and strongly reflects moment ratios such as local ratios of ( $x$ - and $z$-direction-averaged) components of the Reynolds stress tensor. Energetic vortical structures and eruptive motions within a quiescent background turbulent field would certainly make strong contributions to these ratios. In wall flows, those that are parts of quasicyclic processes like bursting events, contribute strongly to the mean strain history, through their large distortion during rapid passage from outer parts of the flow. Motions that are also closely linked with the average local value of the velocity gradient (again $x$ - and $z$-direction averaged) should be influential in determining streak spacing, since the local shear level appears to be a principal requirement for sustaining streak-like behavior. ${ }^{7}$ In the case of wall-bounded turbulent flow, the hairpin vortex has been argued for by a number of researchers ${ }^{34,35}$ as a principal structure associated with streaks. If one considers the action of (near-wall) shear on a vortex line in the $z$ direction, which subsequently distorts into a hairpin vortex (see, for example, Aref and Flinchem ${ }^{36}$ or Smith et al. ${ }^{37}$ ), the evolution of such a structure will certainly change averaged turbulent stress ratios. A companion mechanism of eruption/ sweeping would also have profound effects on the local averaged distortion strain. Thus, such a structure potentially bears a strong identification with the local averaged shear and strain levels, though Sandham and Kleiser ${ }^{38}$ have cautioned that the attention given to this particular structure may be misleading, because of its relatively infrequent occurrence. Similar arguments could be made for the behavior of counter-rotating eddy pairs in shear layers, which have also been proposed as the principal large eddies of near-wall turbulent flow, ${ }^{39,40}$ though little can be said conclusively without more detailed study. The computations of Lee et $a l^{7}{ }^{7}$ suggest that structures that interact with background turbulent motions, as if subjecting them to locally linear distortions, are those that are of importance to the presence and the statistical qualities of streaks. Thus, in principle, to assess the relative importance to streaks of a candidate structure, one might interrogate such structures within databases from numerical simulations of turbulence and assess how strongly and frequently they are identified with high levels of local mean shear, distortion strain, and with linear interaction with neighboring motions.

In conclusion, new experimental measurements of the average spacing of streaks in unsteady turbulent pipe flow have been used to develop a general scaling between streak spacing and a local shear-distortion length scale. This scale appears to be a dominant length scale in streak spacing selection. The "strain-history" approach that revealed this new length scale is significantly different from other attempts to link streak spacing to secondary flows, which result from different local near-wall instabilities. By incorporating the strain history of large eddies in a length scale, a link is made between the weakly strained outer fluid, swept toward the high-shear regions near the wall where streaks are formed, and the ejection/sweep motions that sustain production of new turbulence. The strain history also contains some of the features deemed essential by Waleffe and $\mathrm{Kim}^{13}$ to explaining the mechanism of streak-spacing selection. In the context of their critical Reynolds number $\lambda \vartheta / \nu$ for sustaining the coupled processes of turbulence production, $\sqrt{\langle S\rangle} /\langle\alpha\rangle$ constitutes the local critical velocity scale $\vartheta$ referenced to a local shear velocity, which carries large-scale structural information through the strain-history parameter $\alpha$. For developed flows with qualitatively the same coupled processes of turbulence production, one would also expect this relation between streak spacing and the shear-distortion length scale to hold. Interesting examples that might further confirm the usefulness of this approach include turbulent flow over wavy walls and curved surfaces. 


\section{ACKNOWLEDGMENTS}

The authors gratefully acknowledge the helpful comments and original manuscripts provided by Dr. R. Mankbadi, Dr. M. Maxey, Dr. P. Durbin, and an anonymous reviewer. The second author acknowledges support from the Ministry of Education, Republic of China.

${ }^{1}$ S. J. Kline and P. W. Runstadler, "Some preliminary results of visual studies of the wall layers of the turbulent boundary layers," J. Appl. Mech. 81, Part 3, 166 (1959).

${ }^{2}$ S. J. Kline, W. C. Reynolds, F. A. Schraub, and P. W. Runstadler, "The structure of turbulent boundary layers," J. Fluid Mech. 30, 741 (1967).

${ }^{3}$ M. K. Lee, L. D. Eckelmann, and T. J. Hanratty, "Identification of turbuIent wall eddies through the phase relationship of the components of the fluctuating velocity gradient," J. Fluid Mech. 66, 17 (1974).

${ }^{4} \mathrm{D}$. Oldaker and W. G. Tiederman, "Spatial structure of the viscous sublayer in drag-reducing channel flows," Phys. Fluids 20, S133 (1977).

${ }^{5} \mathrm{H}$. T. Kim, S. J. Kline, and W. C. Reynolds, "The production of turbulence near a smooth wall in a turbulent boundary layer," J. Fluid Mech. 50, 133 (1971).

"J. A. Clark, "A study of incompressible turbulent boundary layers in channel flow," J. Basic Eng. Trans. ASME 90, 455 (1968).

${ }^{7} \mathrm{M}$. J. Lee, J. Kim, and P. Moin, "Structure of turbulence at high shear rate," J. Fluid Mech. 216, 561 (1990).

${ }^{8}$ M. T. Landah1, "On sublayer streaks," J. Fluid Mech. 212, 593 (1990).

${ }^{9} \mathrm{~J}$. Kim, P. Moin, and R. Moser, "Turbulent statistics in fully developed channel flow at low Reynolds number," J. Fluid Mech. 177, 133 (1987).

${ }^{10} \mathrm{G}$. Kawahara, K. Ayukawa, and J. Ochi, "On the origin of streaky structures in wall-bounded turbulent flows," Near-Wall Turbulent Flows, edited by R. M. C. So, C. G. Speziale, and B. E. Launder (Elsevier, New York, 1993), p. 403.

${ }^{11}$ P. S. Jang, D. J. Benney, and R. L. Gran, "On the origin of streamwise vortices in a turbulent boundary layer," J. Fluid Mech. 169, 109 (1986).

${ }^{12} \mathrm{D}$. J. Benney and L. H. Gustavsson, "A new mechanism for linear and nonlinear hydrodynamic stability," Stud. Appl. Math. 64, 185 (1981).

${ }^{13} \mathrm{~F}$. Waleffe and J. Kim, "On the origin of streaks in turbulent shear flows," Proceedings of the 8th Symposium on Turbulent Shear Flows, Munich, 1991.

${ }^{14} \mathrm{~T}$. Sarpkaya, "On the instability of the Stokes boundary layer," Near-Wall Turbulent Flows, edited by R. M. C. So, C. G. Spezilale, and B. E. Launder (Elsevier, New York, 1993), p. 479.

${ }^{15} \mathrm{~J}$.-L Hwang and G. J. Brereton, "Turbulence in high-frequency periodic fully developed pipe flow," Turbulent Shear Flows 8, edited by F. Durst, B. E. Launder, F. W. Schmidt, U. Schumann, and J. H. Whitelaw (Springer, Berlin, 1993), p. 431.

${ }^{16} \mathrm{~S}$. Tardu and G. Binder, "Ejections and bursts in pulsatile turbulent wall flow measurements and visualizations," Proceedings of the 7th Symposium on Turbulent Shear Flows, Stanford University, Stanford, CA, 1989.

${ }^{17}$ G. J. Brereton and W. C. Reynolds, "Dynamic response of boundary layer turbulence to oscillatory shear," Phys. Fluids A 3, 178 (1991).

${ }^{18} \mathrm{~J}$.-L. Hwang, "An experimental study of the fluid mechanics of turbulent pipe flow when subjected to forced oscillation at high frequencies," Ph.D. thesis, The University of Michigan, Ann Arbor, Michigan, 1992.

${ }^{19}$ T. S. Luchik and W. G. Tiederman, "Timescale and structure of ejections and bursts in turbulent channel flows," J. Fluid Mech. 174, 529 (1987).

${ }^{20} \mathrm{~L}$. Shemer, E. Kit, and I. Wygnanski, "On the impedance of the pipe in laminar and turbulent pulsating flows," Exp. Fluids 3, 185 (1985).

${ }^{21} \Lambda$. K. M. F. Hussein and W. C. Rcynolds, "The mechanics of an organized wave in turbulent shear flow," J. Fluid Mech. 41, 241 (1970).

${ }^{32}$ F. A. Schraub, S. J. Kline, J. Henry, P. W. Runstadler, and A. Littell, "Use of hydrogen bubbles for quantitative determination of time-dependent velocity fields in low-speed water flows," J. Basic Eng. Trans. ASME 87, 429 (1965).

${ }^{23} \mathrm{~B}$. V. Achia and D. W. Thompson, "Structure of the turbulent boundary layer in drag-reducing pipe flow," J. Fluid Mech. 81, 439 (1976).

${ }^{24} \mathrm{C}$. R. Smith and S. P. Metzler, "'The characteristics of low-speed streaks in the near-wall region of a turbulent boundary layer," J. Fluid Mech. 129, 27 (1983).

${ }^{25} \mathrm{G}$. J. Brereton, "Deduction of skin friction by Clauser technique in unsteady turbulent boundary layers," Exp. Fluids. 7, 422 (1989).

${ }^{26} \mathrm{~T}$. Sarpkaya, "Coherent structures in oscillatory boundary layers," J. Fluid Mech. 253, 105 (1993).

${ }^{27}$ M. J. Lee, J. Kim, and P. Moin, "Turbulence structure at high shear rate," Proceedings of the 6th Symposium on Turbulent Shear Flows, Toulouse, France, 1987, p. 22.6.1.

${ }^{28} \mathrm{~A}$. A. Townsend, "Entrainment and the structure of turbulent flow," J. Fluid Mech: 41, 13 (1970).

${ }^{29}$ A. A. Townsend, The Structure of Turbulent Shear Flow' (Cambridge U.P., Cambridge, 1976).

${ }^{30} \mathrm{~A}$. $\mathrm{A}$. Townsend, "The responsc of shcarcd turbulence to additional distortion," J. Fluid Mech. 98, 171 (1980).

${ }^{31}$ M. R. Maxey, "Distortion of turbulence in flows with parallel streamlines," J. Fluid Mech. 124, 261 (1982).

${ }^{32}$ N. Kasagi, "Structural study of near-wall turbulence and its heat transfer mechanism," Near-Wall Turbulence: 1988 Zoran Zaric Memorial Conference, edited by S. J. Kline and N. H. Afgan (Hemisphere, New York, 1990), p. 596.

${ }^{33} \mathrm{M}$. J. Lee and J. C. R. Hunt "The structure of sheared turbulence near a plane boundary," in Ref. 16.

${ }^{34}$ T. Theodorsen, "Mechanism of turbulence," in Proceedings of the 2nd Midwestern Conference on Fluid Mechanics, Ohio State University, Columbus, Ohio, 1952.

${ }^{35} \mathrm{M}$. R. Head and P. Bandyopadhyay, "New aspects of turbulent boundary layer structure," J. Fluid Mech. 107, 297 (1981).

${ }^{36} \mathrm{H}$. Aref and E. P. Flinchem, "Dynamics of a vortex filament in a shear flow," J. Fluid Mech. 148, 477 (1984).

${ }^{37}$ C. R. Smith, J. D. A. Walker, A. H. Haidari, and U. Sobrun, "On the dynamics of near-wall turbulence." Philos. Trans. R. Soc. London Ser. A 336, 131 (1991).

${ }^{38}$ N. D. Sandham and L. Kleiser, "The late stages of transition to turbulence in channel flow," J. Fluid Mech. 245, 319 (1992).

${ }^{39}$ P. Bakewell and J. L. Lumley, "Viscous sublayer and adjacent wall region in turbulent pipe flow," Phys. Fluids 10, 1880 (1967).

${ }^{40} \mathrm{R}$. F. Blackwelder and H. Eckelmann, "Streamwise vortices associated with the bursting phenomenon," J. Fluid Mech. 94, 577 (1979). 